\title{
Coefficient Estimates for a New Subclass of Analytic and Bi-Univalent Functions Defined by Hadamard Product
}

\author{
Serap Bulut \\ Civil Aviation College, Kocaeli University, Arslanbey Campus, 41285 İzmit, Kocaeli, Turkey \\ Correspondence should be addressed to Serap Bulut; bulutserap@yahoo.com \\ Received 19 July 2014; Accepted 10 October 2014; Published 10 November 2014 \\ Academic Editor: Jacek Dziok
}

Copyright (C) 2014 Serap Bulut. This is an open access article distributed under the Creative Commons Attribution License, which permits unrestricted use, distribution, and reproduction in any medium, provided the original work is properly cited.

We introduce and investigate a new general subclass $\mathscr{H}_{\Sigma}^{\lambda, \mu}(\varphi ; \Theta)$ of analytic and bi-univalent functions in the open unit disk $\mathbb{U}$. For functions belonging to this class, we obtain estimates on the first two Taylor-Maclaurin coefficients $\left|a_{2}\right|$ and $\left|a_{3}\right|$.

\section{Introduction}

Let $\mathscr{A}$ denote the class of all functions of the form

$$
f(z)=z+\sum_{n=2}^{\infty} a_{n} z^{n}
$$

which are analytic in the open unit disk

$$
\mathbb{U}=\{z: z \in \mathbb{C},|z|<1\} .
$$

We also denote by $\mathcal{S}$ the class of all functions in the normalized analytic function class $\mathscr{A}$ which are univalent in $\mathbb{U}$.

Since univalent functions are one-to-one, they are invertible and the inverse functions need not be defined on the entire unit disk $\mathbb{U}$. In fact, the Koebe one-quarter theorem [1] ensures that the image of $\mathbb{U}$ under every univalent function $f \in \delta$ contains a disk of radius $1 / 4$. Thus every function $f \in \mathscr{A}$ has an inverse $f^{-1}$, which is defined by

$$
\begin{gathered}
f^{-1}(f(z))=z \quad(z \in \mathbb{U}), \\
f\left(f^{-1}(w)\right)=w \quad\left(|w|<r_{0}(f) ; r_{0}(f) \geq \frac{1}{4}\right) .
\end{gathered}
$$

In fact, the inverse function $f^{-1}$ is given by

$$
\begin{aligned}
f^{-1}(w)= & w-a_{2} w^{2}+\left(2 a_{2}^{2}-a_{3}\right) w^{3} \\
& -\left(5 a_{2}^{3}-5 a_{2} a_{3}+a_{4}\right) w^{4}+\cdots .
\end{aligned}
$$

Denote by $f * \Theta$ the Hadamard product (or convolution) of the functions $f$ and $\Theta$; that is, if $f$ is given by (1) and $\Theta$ is given by

$$
\Theta(z)=z+\sum_{n=2}^{\infty} b_{n} z^{n} \quad\left(b_{n}>0\right),
$$

then

$$
(f * \Theta)(z)=z+\sum_{n=2}^{\infty} a_{n} b_{n} z^{n} .
$$

For two functions $f$ and $\Theta$, analytic in $\mathbb{U}$, we say that the function $f$ is subordinate to $\Theta$ in $\mathbb{U}$ and write

$$
f(z)<\Theta(z) \quad(z \in \mathbb{U}),
$$

if there exists a Schwarz function $\omega$, which is analytic in $\mathbb{U}$ with

$$
\omega(0)=0, \quad|\omega(z)|<1 \quad(z \in \mathbb{U})
$$

such that

$$
f(z)=\Theta(\omega(z)) \quad(z \in \mathbb{U}) .
$$

Indeed, it is known that

$$
\begin{gathered}
f(z) \prec \Theta(z) \\
(z \in \mathbb{U}) \Longrightarrow f(0)=\Theta(0), \quad f(\mathbb{U}) \subset \Theta(\mathbb{U}) .
\end{gathered}
$$


Furthermore, if the function $\Theta$ is univalent in $\mathbb{U}$, then we have the following equivalence:

$$
\begin{gathered}
f(z) \prec \Theta(z) \\
(z \in \mathbb{U}) \Longleftrightarrow f(0)=\Theta(0), \quad f(\mathbb{U}) \subset \Theta(\mathbb{U}) .
\end{gathered}
$$

A function $f \in \mathscr{A}$ is said to be bi-univalent in $\mathbb{U}$ if both $f$ and $f^{-1}$ are univalent in $\mathbb{U}$. Let $\Sigma$ denote the class of bi-univalent functions in $\mathbb{U}$ given by (1). For a brief history and interesting examples of functions in the class $\Sigma$, see [2] (see also [3]). In fact, the aforecited work of Srivastava et al. [2] essentially revived the investigation of various subclasses of the bi-univalent function class $\Sigma$ in recent years; it was followed by such works as those by Tang et al. [4], El-Ashwah [5], Frasin and Aouf [6], Aouf et al. [7], and others (see, e.g., $[2,8-15])$.

Throughout this paper, we assume that $\varphi$ is an analytic function with positive real part in the unit disk $\mathbb{U}$, satisfying $\varphi(0)=1, \varphi^{\prime}(0)>0$, and $\varphi(\mathbb{U})$ is symmetric with respect to the real axis. Such a function has a series expansion of the form

$$
\varphi(z)=1+B_{1} z+B_{2} z^{2}+\cdots, \quad\left(B_{1}>0\right) .
$$

With this assumption on $\varphi$, we now introduce the following subclass of bi-univalent functions.

Definition 1. Let the function $f$, defined by (1), be in the analytic function class $\mathscr{A}$ and let $\Theta \in \Sigma$. We say that

$$
f \in \mathscr{H}_{\Sigma}^{\lambda, \mu}(\varphi ; \Theta) \quad(\lambda \geq 1, \mu \geq 0)
$$

if the following conditions are satisfied:

$$
\begin{gathered}
f \in \Sigma \\
(1-\lambda)\left(\frac{(f * \Theta)(z)}{z}\right)^{\mu}+\lambda(f * \Theta)^{\prime}(z)\left(\frac{(f * \Theta)(z)}{z}\right)^{\mu-1} \\
\prec \varphi(z) \quad(z \in \mathbb{U}), \\
(1-\lambda)\left(\frac{(f * \Theta)^{-1}(w)}{w}\right)^{\mu}+\lambda\left((f * \Theta)^{-1}\right)^{\prime}(w) \\
\times\left(\frac{(f * \Theta)^{-1}(w)}{w}\right)^{\mu-1} \prec \varphi(w) \quad(w \in \mathbb{U})
\end{gathered}
$$

where the function $(f * \Theta)^{-1}$ is given by

$$
\begin{aligned}
(f * \Theta)^{-1}(w)= & w-a_{2} b_{2} w^{2}+\left(2 a_{2}^{2} b_{2}^{2}-a_{3} b_{3}\right) w^{3} \\
& -\left(5 a_{2}^{3} b_{2}^{3}-5 a_{2} b_{2} a_{3} b_{3}+a_{4} b_{4}\right) w^{4}+\cdots
\end{aligned}
$$

Remark 2. If we let

$$
\Theta(z)=\frac{z}{1-z},
$$

then the class $\mathscr{H}_{\Sigma}^{\lambda, \mu}(\varphi ; \Theta)$ reduces to the class denoted by $\mathscr{H}_{\Sigma}^{\mu}(\lambda, \varphi)$ which is the subclass of the functions $f \in \Sigma$ satisfying

$$
\begin{aligned}
& (1-\lambda)\left(\frac{f(z)}{z}\right)^{\mu}+\lambda f^{\prime}(z)\left(\frac{f(z)}{z}\right)^{\mu-1} \prec \varphi(z), \\
& (1-\lambda)\left(\frac{g(w)}{w}\right)^{\mu}+\lambda g^{\prime}(w)\left(\frac{g(w)}{w}\right)^{\mu-1} \prec \varphi(w),
\end{aligned}
$$

where the function $g$ is defined by

$$
\begin{aligned}
g(w)= & w-a_{2} w^{2}+\left(2 a_{2}^{2}-a_{3}\right) w^{3} \\
& -\left(5 a_{2}^{3}-5 a_{2} a_{3}+a_{4}\right) w^{4}+\cdots,
\end{aligned}
$$

which was introduced and studied recently by Tang et al. [4].

Remark 3. If we let

$$
\varphi(z)=\left(\frac{1+z}{1-z}\right)^{\alpha} \quad(0<\alpha \leq 1),
$$

then the class $\mathscr{H}_{\Sigma}^{\lambda, \mu}(\varphi ; \Theta)$ reduces to the new class denoted by $\mathscr{H}_{\Sigma}^{\lambda, \mu}(\alpha ; \Theta)$ which is the subclass of the functions $f \in \Sigma$ satisfying

$$
\begin{aligned}
\mid \arg \{ & (1-\lambda)\left(\frac{(f * \Theta)(z)}{z}\right)^{\mu} \\
& \left.+\lambda(f * \Theta)^{\prime}(z)\left(\frac{(f * \Theta)(z)}{z}\right)^{\mu-1}\right\} \mid<\frac{\alpha \pi}{2}, \\
\mid \arg \left\{(1-\lambda)\left(\frac{(f * \Theta)^{-1}(w)}{w}\right)^{\mu}\right. & \left\{\lambda\left((f * \Theta)^{-1}\right)^{\prime}(w)\left(\frac{(f * \Theta)^{-1}(w)}{w}\right)^{\mu-1}\right\} \mid<\frac{\alpha \pi}{2},
\end{aligned}
$$

where the function $(f * \Theta)^{-1}$ is defined by (15). Also we have the following classes:

(i)

$$
\mathscr{H}_{\Sigma}^{\lambda, 1}(\alpha ; \Theta)=\mathscr{B}_{\Sigma}(\Theta, \alpha, \lambda)
$$

introduced and studied recently by El-Ashwah [5];

(ii)

$$
\mathscr{H}_{\Sigma}^{\lambda, \mu}\left(\alpha ; \frac{z}{1-z}\right)=\mathcal{N}_{\Sigma}^{\mu}(\alpha, \lambda)
$$

introduced and studied recently by Çağlar et al. [10]; 
(iii)

$$
\mathscr{H}_{\Sigma}^{\lambda, 1}\left(\alpha ; \frac{z}{1-z}\right)=\mathscr{B}_{\Sigma}(\alpha, \lambda)
$$

introduced and studied by Frasin and Aouf [6];

(iv)

$$
\mathscr{H}_{\Sigma}^{1,0}\left(\alpha ; \frac{z}{1-z}\right)=\mathcal{S}_{\Sigma}^{*}[\alpha]
$$

of strongly bi-starlike functions of order $\alpha(0<\alpha \leq 1)$ introduced and studied by Brannan and Taha [3];

(v)

$$
\mathscr{H}_{\Sigma}^{\lambda, 1}\left(\alpha ; z+\sum_{n=2}^{\infty} \frac{\left(a_{1}\right)_{n} \cdots\left(a_{q}\right)_{n}}{\left(b_{1}\right)_{n} \cdots\left(b_{s}\right)_{n}} \frac{1}{n !} z^{n}\right)=\mathscr{T}_{q, s}^{\Sigma}\left[a_{1} ; b_{1}, \alpha, \lambda\right]
$$

introduced and studied recently by Aouf et al. [7].

Remark 4. If we let

$$
\varphi(z)=\frac{1+(1-2 \beta) z}{1-z} \quad(0 \leq \beta<1),
$$

then the class $\mathscr{H}_{\Sigma}^{\lambda, \mu}(\varphi ; \Theta)$ reduces to the new class denoted by $\mathscr{H}_{\Sigma}^{\lambda, \mu}(\beta ; \Theta)$ which is the subclass of the functions $f \in \Sigma$ satisfying

$$
\begin{aligned}
& \Re\left\{(1-\lambda)\left(\frac{(f * \Theta)(z)}{z}\right)^{\mu}\right. \\
& \left.+\lambda(f * \Theta)^{\prime}(z)\left(\frac{(f * \Theta)(z)}{z}\right)^{\mu-1}\right\}>\beta, \\
& \Re\left\{(1-\lambda)\left(\frac{(f * \Theta)^{-1}(w)}{w}\right)^{\mu}\right. \\
& \left.+\lambda\left((f * \Theta)^{-1}\right)^{\prime}(w)\left(\frac{(f * \Theta)^{-1}(w)}{w}\right)^{\mu-1}\right\}>\beta,
\end{aligned}
$$

where the function $(f * \Theta)^{-1}$ is defined by (15). Also we have the following classes:

(i)

$$
\mathscr{H}_{\Sigma}^{\lambda, 1}(\beta ; \Theta)=\mathscr{B}_{\Sigma}(\Theta, \beta, \lambda)
$$

introduced and studied recently by El-Ashwah [5];

(ii)

$$
\mathscr{H}_{\Sigma}^{\lambda, \mu}\left(\beta ; \frac{z}{1-z}\right)=\mathcal{N}_{\Sigma}^{\mu}(\beta, \lambda)
$$

introduced and studied recently by Çağlar et al. [10]; (iii)

$$
\mathscr{H}_{\Sigma}^{\lambda, 1}\left(\beta ; \frac{z}{1-z}\right)=\mathscr{B}_{\Sigma}(\beta, \lambda)
$$

introduced and studied by Frasin and Aouf [6];

(iv)

$$
\mathscr{H}_{\Sigma}^{1,0}\left(\beta ; \frac{z}{1-z}\right)=\mathcal{S}_{\Sigma}^{*}(\beta)
$$

of bi-starlike functions of order $\beta(0 \leq \beta<1)$ introduced and studied by Brannan and Taha [3];

(v)

$$
\mathscr{H}_{\Sigma}^{\lambda, 1}\left(\alpha ; z+\sum_{n=2}^{\infty} \frac{\left(a_{1}\right)_{n} \cdots\left(a_{q}\right)_{n}}{\left(b_{1}\right)_{n} \cdots\left(b_{s}\right)_{n}} \frac{1}{n !} z^{n}\right)=\mathscr{T}_{q, s}^{\Sigma}\left[a_{1} ; b_{1}, \beta, \lambda\right]
$$

introduced and studied recently by Aouf et al. [7].

For more results see also [2, 8, 13, 15-17].

Firstly, in order to derive our main results, we need to go to following lemma.

Lemma 5 (see [18]). If $p \in \mathscr{P}$, then $\left|c_{n}\right| \leq 2$ for each $n$, where $\mathscr{P}$ is the family of all functions $p$ analytic in $\mathbb{U}$ for which

$$
\Re(p(z))>0, \quad p(z)=1+c_{1} z+c_{2} z^{2}+\cdots
$$

for $z \in \mathbb{U}$.

\section{A Set of General Coefficient Estimates}

In this section, we state and prove our general results involving the bi-univalent function class $\mathscr{H}_{\Sigma}^{\lambda, \mu}(\varphi ; \Theta)$ given by Definition 1.

Theorem 6. Let the function $f(z)$ given by the TaylorMaclaurin series expansion (1) be in the function class

$$
\mathscr{H}_{\Sigma}^{\lambda, \mu}(\varphi ; \Theta) \quad(\lambda \geq 1, \mu \geq 0)
$$

with

$$
\Theta \in \Sigma, \quad \Theta(z)=z+\sum_{n=2}^{\infty} b_{n} z^{n} \quad\left(b_{n}>0\right) .
$$


Then

$\left|a_{2}\right|$

$$
\begin{aligned}
\leq \frac{1}{b_{2}} \min \left\{\frac{B_{1}}{\lambda+\mu}, \sqrt{\frac{2\left(B_{1}+\left|B_{2}-B_{1}\right|\right)}{(\mu+1)(2 \lambda+\mu)}}\right. \\
\\
\\
\left.\frac{B_{1} \sqrt{2 B_{1}}}{\sqrt{\left|B_{1}^{2}(\mu+1)(2 \lambda+\mu)-2\left(B_{2}-B_{1}\right)(\lambda+\mu)^{2}\right|}}\right\},
\end{aligned}
$$

$$
\begin{aligned}
\left|a_{3}\right| \leq \frac{1}{b_{3}} \min \{ & \frac{B_{1}^{2}}{(\lambda+\mu)^{2}}+\frac{B_{1}}{2 \lambda+\mu}, \\
& \frac{B_{1}[(\mu+3)+|1-\mu|]}{2(\mu+1)(2 \lambda+\mu)}+\frac{2\left|B_{2}-B_{1}\right|}{(\mu+1)(2 \lambda+\mu)}, \\
& \frac{2 B_{1}^{3}}{\left|B_{1}^{2}(\mu+1)(2 \lambda+\mu)-2\left(B_{2}-B_{1}\right)(\lambda+\mu)^{2}\right|} \\
& \left.+\frac{B_{1}}{2 \lambda+\mu}\right\} .
\end{aligned}
$$

Proof. Let $f \in \mathscr{H}_{\Sigma}^{\lambda, \mu}(\varphi ; \Theta)$. Then there are analytic functions $u, v: \mathbb{U} \rightarrow \mathbb{U}$, with $u(0)=v(0)=0$, satisfying

$$
\begin{aligned}
& (1-\lambda)\left(\frac{(f * \Theta)(z)}{z}\right)^{\mu}+\lambda(f * \Theta)^{\prime}(z)\left(\frac{(f * \Theta)(z)}{z}\right)^{\mu-1} \\
& =\varphi(u(z)) \\
& (1-\lambda)\left(\frac{(f * \Theta)^{-1}(w)}{w}\right)^{\mu} \\
& +\lambda\left((f * \Theta)^{-1}\right)^{\prime}(w)\left(\frac{(f * \Theta)^{-1}(w)}{w}\right)^{\mu-1}=\varphi(v(w)) .
\end{aligned}
$$

Define the functions $p$ and $q$ by

$$
\begin{aligned}
& p(z)=\frac{1+u(z)}{1-u(z)}=1+p_{1} z+p_{2} z^{2}+\cdots \\
& q(z)=\frac{1+v(z)}{1-v(z)}=1+q_{1} z+q_{2} z^{2}+\cdots
\end{aligned}
$$

or, equivalently,

$$
\begin{aligned}
& u(z)=\frac{p(z)-1}{p(z)+1}=\frac{1}{2}\left[p_{1} z+\left(p_{2}-\frac{p_{1}^{2}}{2}\right) z^{2}+\cdots\right], \\
& v(z)=\frac{q(z)-1}{q(z)+1}=\frac{1}{2}\left[q_{1} z+\left(q_{2}-\frac{q_{1}^{2}}{2}\right) z^{2}+\cdots\right] .
\end{aligned}
$$

Then $p$ and $q$ are analytic in $\mathbb{U}$ with $p(0)=1=q(0)$. Since $u, v: \mathbb{U} \rightarrow \mathbb{U}$, the functions $p$ and $q$ have a positive real part in $\mathbb{U}$, and by Lemma $5\left|p_{n}\right| \leq 2$ and $\left|q_{n}\right| \leq 2$ for each $n$. Also it is clear that

$$
\begin{aligned}
& (1-\lambda)\left(\frac{(f * \Theta)(z)}{z}\right)^{\mu}+\lambda(f * \Theta)^{\prime}(z)\left(\frac{(f * \Theta)(z)}{z}\right)^{\mu-1} \\
& =\varphi\left(\frac{p(z)-1}{p(z)+1}\right) \\
& (1-\lambda)\left(\frac{(f * \Theta)^{-1}(w)}{w}\right)^{\mu}+\lambda\left((f * \Theta)^{-1}\right)^{\prime}(w) \\
& \quad \times\left(\frac{(f * \Theta)^{-1}(w)}{w}\right)^{\mu-1}=\varphi\left(\frac{q(w)-1}{q(w)+1}\right) .
\end{aligned}
$$

Using (40) and (41) together with (12), we get

$$
\begin{aligned}
& \varphi\left(\frac{p(z)-1}{p(z)+1}\right) \\
& \quad=1+\frac{1}{2} B_{1} p_{1} z+\left(\frac{1}{2} B_{1}\left(p_{2}-\frac{p_{1}^{2}}{2}\right)+\frac{1}{4} B_{2} p_{1}^{2}\right) z^{2}+\cdots, \\
& \varphi\left(\frac{q(w)-1}{q(w)+1}\right) \\
& \quad=1+\frac{1}{2} B_{1} q_{1} w+\left(\frac{1}{2} B_{1}\left(q_{2}-\frac{q_{1}^{2}}{2}\right)+\frac{1}{4} B_{2} q_{1}^{2}\right) w^{2}+\cdots
\end{aligned}
$$

Now, upon equating the coefficients in (42)-(43), we get

$$
\begin{gathered}
(\lambda+\mu) b_{2} a_{2}=\frac{1}{2} B_{1} p_{1}, \\
(2 \lambda+\mu) b_{3} a_{3}+(\mu-1)\left(\lambda+\frac{\mu}{2}\right) b_{2}^{2} a_{2}^{2} \\
=\frac{1}{2} B_{1}\left(p_{2}-\frac{p_{1}^{2}}{2}\right)+\frac{1}{4} B_{2} p_{1}^{2}, \\
-(\lambda+\mu) b_{2} a_{2}=\frac{1}{2} B_{1} q_{1}, \\
-(2 \lambda+\mu) b_{3} a_{3}+(\mu+3)\left(\lambda+\frac{\mu}{2}\right) b_{2}^{2} a_{2}^{2} \\
=\frac{1}{2} B_{1}\left(q_{2}-\frac{q_{1}^{2}}{2}\right)+\frac{1}{4} B_{2} q_{1}^{2} .
\end{gathered}
$$

From (44) and (46), it follows that

$$
\begin{aligned}
p_{1} & =-q_{1}, \\
2(\lambda+\mu)^{2} b_{2}^{2} a_{2}^{2} & =\frac{1}{4} B_{1}^{2}\left(p_{1}^{2}+q_{1}^{2}\right) .
\end{aligned}
$$


Now (45), (47), and (48) yield

$$
a_{2}^{2}=\frac{B_{1}\left(p_{2}+q_{2}\right)+\left(B_{2}-B_{1}\right) p_{1}^{2}}{2(\mu+1)(2 \lambda+\mu) b_{2}^{2}} .
$$

Also from (45), (47), and (49), we get

$$
a_{2}^{2}=\frac{B_{1}^{3}\left(p_{2}+q_{2}\right)}{2\left[B_{1}^{2}(\mu+1)(2 \lambda+\mu)-2\left(B_{2}-B_{1}\right)(\lambda+\mu)^{2}\right] b_{2}^{2}} .
$$

Applying Lemma 5 for (44), (50), and (51), we get the desired estimate on the coefficient $\left|a_{2}\right|$ as asserted in (36).

Next, in order to find the bound on the coefficient $\left|a_{3}\right|$, by subtracting (47) from (45), we obtain

$$
2(2 \lambda+\mu) b_{3} a_{3}-2(2 \lambda+\mu) b_{2}^{2} a_{2}^{2}=\frac{1}{2} B_{1}\left(p_{2}-q_{2}\right) .
$$

Upon substituting the value of $a_{2}^{2}$ from (44), (50), and (51) into (52), it follows that

$$
\begin{gathered}
a_{3}=\frac{B_{1}^{2} p_{1}^{2}}{4(\lambda+\mu)^{2} b_{3}}+\frac{B_{1}\left(p_{2}-q_{2}\right)}{4(2 \lambda+\mu) b_{3}} \\
a_{3}=\frac{B_{1}\left(p_{2}+q_{2}\right)+\left(B_{2}-B_{1}\right) p_{1}^{2}}{2(\mu+1)(2 \lambda+\mu) b_{3}}+\frac{B_{1}\left(p_{2}-q_{2}\right)}{4(2 \lambda+\mu) b_{3}} \\
=\frac{B_{1}\left[(\mu+3) p_{2}+(1-\mu) q_{2}\right]+2\left(B_{2}-B_{1}\right) p_{1}^{2}}{4(\mu+1)(2 \lambda+\mu) b_{3}} \\
a_{3}=\frac{B_{1}^{3}\left(p_{2}+q_{2}\right)}{2\left[B_{1}^{2}(\mu+1)(2 \lambda+\mu)-2\left(B_{2}-B_{1}\right)(\lambda+\mu)^{2}\right] b_{3}} \\
+\frac{B_{1}\left(p_{2}-q_{2}\right)}{4(2 \lambda+\mu) b_{3}},
\end{gathered}
$$

respectively. Applying Lemma 5 for (53), (54), and (55), we get the desired estimate on the coefficient $\left|a_{3}\right|$ as asserted in (37).

If we take

$$
\Theta(z)=\frac{z}{1-z}
$$

in Theorem 6, then we have the following corollary.

Corollary 7. Let the function $f(z)$ given by the TaylorMaclaurin series expansion (1) be in the function class

$$
\mathscr{H}_{\Sigma}^{\mu}(\lambda, \varphi) \quad(\lambda \geq 1, \mu \geq 0) .
$$

Then

$$
\left|a_{2}\right|
$$

$$
\begin{aligned}
& \leq \min \left\{\frac{B_{1}}{\lambda+\mu}, \sqrt{\frac{2\left(B_{1}+\left|B_{2}-B_{1}\right|\right)}{(\mu+1)(2 \lambda+\mu)}},\right. \\
& \left.\frac{B_{1} \sqrt{2 B_{1}}}{\sqrt{\left|B_{1}^{2}(\mu+1)(2 \lambda+\mu)-2\left(B_{2}-B_{1}\right)(\lambda+\mu)^{2}\right|}}\right\} \text {, } \\
& \left|a_{3}\right| \leq \min \left\{\frac{B_{1}^{2}}{(\lambda+\mu)^{2}}+\frac{B_{1}}{2 \lambda+\mu},\right. \\
& \frac{B_{1}[(\mu+3)+|1-\mu|]}{2(\mu+1)(2 \lambda+\mu)}+\frac{2\left|B_{2}-B_{1}\right|}{(\mu+1)(2 \lambda+\mu)}, \\
& \frac{2 B_{1}^{3}}{\left|B_{1}^{2}(\mu+1)(2 \lambda+\mu)-2\left(B_{2}-B_{1}\right)(\lambda+\mu)^{2}\right|} \\
& \left.+\frac{B_{1}}{2 \lambda+\mu}\right\} \text {. }
\end{aligned}
$$

Remark 8. Corollary 7 is an improvement of the estimates which were given by Tang et al. [4, Theorem 2.1].

Remark 9. If we set $\lambda=1$ and $\mu=1$ in Corollary 7, then we have an improvement of the estimates which were given by Ali et al. [16, Theorem 2.1].

If we take

$$
\varphi(z)=\left(\frac{1+z}{1-z}\right)^{\alpha} \quad(0<\alpha \leq 1)
$$

in Theorem 6, then we have the following corollary.

Corollary 10. Let the function $f(z)$ given by the TaylorMaclaurin series expansion (1) be in the function class

$$
\mathscr{H}_{\Sigma}^{\lambda, \mu}(\alpha ; \Theta) \quad(\lambda \geq 1, \mu \geq 0,0<\alpha \leq 1)
$$

with

$$
\Theta \in \Sigma, \quad \Theta(z)=z+\sum_{n=2}^{\infty} b_{n} z^{n} \quad\left(b_{n}>0\right) .
$$


Then

$$
\begin{aligned}
& \left|a_{2}\right| \leq \frac{1}{b_{2}} \min \left\{\frac{2 \alpha}{\lambda+\mu}, \sqrt{\frac{4 \alpha(2-\alpha)}{(\mu+1)(2 \lambda+\mu)}},\right. \\
& \left.\frac{2 \alpha}{\sqrt{(\lambda+\mu)^{2}+\alpha\left(\mu+2 \lambda-\lambda^{2}\right)}}\right\}, \\
& \left|a_{3}\right| \leq \frac{1}{b_{3}} \min \left\{\frac{4 \alpha^{2}}{(\lambda+\mu)^{2}}+\frac{2 \alpha}{2 \lambda+\mu},\right. \\
& \frac{2 \alpha[(\mu+3)+|1-\mu|]}{2(\mu+1)(2 \lambda+\mu)}+\frac{4 \alpha(1-\alpha)}{(\mu+1)(2 \lambda+\mu)}, \\
& \left.\frac{4 \alpha^{2}}{(\lambda+\mu)^{2}+\alpha\left(\mu+2 \lambda-\lambda^{2}\right)}+\frac{2 \alpha}{2 \lambda+\mu}\right\} \text {. }
\end{aligned}
$$

Remark 11. In Corollary 10,

(i) if we set $\mu=1$, then we have an improvement of the estimates which were given by El-Ashwah [5, Theorem 1];

(ii) if we set $\Theta(z)=z /(1-z)$, then we have an improvement of the estimates which were given by Çağlar et al. [10, Theorem 2.1];

(iii) if we set $\Theta(z)=z /(1-z)$ and $\mu=1$, then we have an improvement of the estimates which were given by Frasin and Aouf [6, Theorem 2.2];

(iv) if we set $\Theta(z)=z+\sum_{n=2}^{\infty}\left(\left(\left(a_{1}\right)_{n} \cdots\left(a_{q}\right)_{n} /\left(b_{1}\right)_{n} \cdots\right.\right.$ $\left.\left.\left(b_{s}\right)_{n}\right)(1 / n !) z^{n}\right)$ and $\mu=1$, then we have an improvement of the estimates which were given by Aouf et al. [7, Theorem 4].

If we take

$$
\varphi(z)=\frac{1+(1-2 \beta) z}{1-z} \quad(0 \leq \beta<1)
$$

in Theorem 6, then we have the following corollary.

Corollary 12. Let the function $f(z)$ given by the TaylorMaclaurin series expansion (1) be in the function class

$$
\mathscr{B}_{\Sigma}(\Theta, \beta, \lambda) \quad(\lambda \geq 1,0 \leq \beta<1)
$$

with

$$
\Theta \in \Sigma, \quad \Theta(z)=z+\sum_{n=2}^{\infty} b_{n} z^{n} \quad\left(b_{n}>0\right) .
$$

Then

$$
\begin{aligned}
& \left|a_{2}\right| \leq \frac{1}{b_{2}} \min \left\{\frac{2(1-\beta)}{\lambda+\mu}, \sqrt{\left.\frac{4(1-\beta)}{(\mu+1)(2 \lambda+\mu)}\right\},}\right. \\
& \left|a_{3}\right| \\
& \leq\left\{\begin{array}{l}
\frac{1}{b_{3}} \min \left\{\frac{4(1-\beta)^{2}}{(\lambda+\mu)^{2}}+\frac{2(1-\beta)}{2 \lambda+\mu}, \frac{4(1-\beta)}{(\mu+1)(2 \lambda+\mu)}\right\}, \\
0 \leq \mu<1, \\
\frac{2(1-\beta)}{(2 \lambda+\mu) b_{3}}, \\
\mu \geq 1 .
\end{array}\right.
\end{aligned}
$$

\section{Remark 13. In Corollary 12,}

(i) if we set $\mu=1$, then we have an improvement of the estimates which were given by El-Ashwah [5, Theorem 2];

(ii) if we set $\Theta(z)=z /(1-z)$, then we have the estimates which were given by Çağlar et al. [10, Theorem 3.1];

(iii) if we set $\Theta(z)=z /(1-z)$ and $\mu=1$, then we have an improvement of the estimates which were given by Frasin and Aouf [6, Theorem 3.2];

(iv) if we set $\Theta(z)=z+\sum_{n=2}^{\infty}\left(\left(\left(a_{1}\right)_{n} \cdots\left(a_{q}\right)_{n} /\left(b_{1}\right)_{n} \cdots\right.\right.$ $\left.\left.\left(b_{s}\right)_{n}\right)(1 / n !) z^{n}\right)$ and $\mu=1$, then we have an improvement of the estimates which were given by Aouf et al. [7, Theorem 8].

\section{Conflict of Interests}

The author declares that there is no conflict of interests regarding the publication of this paper.

\section{References}

[1] P. L. Duren, Univalent Functions, vol. 259 of Grundlehren der Mathematischen Wissenschaften, Springer, New York, NY, USA, 1983.

[2] H. M. Srivastava, A. K. Mishra, and P. Gochhayat, "Certain subclasses of analytic and bi-univalent functions," Applied Mathematics Letters, vol. 23, no. 10, pp. 1188-1192, 2010.

[3] D. A. Brannan and T. S. Taha, "On some classes of bi-univalent functions," in Mathematical Analysis and Its Applications, S. M. Mazhar, A. Hamoui, and N. S. Faour, Eds., vol. 3 of KFAS Proceedings Series, pp. 53-60, Pergamon Press (Elsevier Science), 1988, Studia Universitatis Babes-Bolyai, Series Mathematica, vol. 31, no. 2, 70-77, 1986.

[4] H. Tang, G.-T. Deng, and S.-H. Li, "Coefficient estimates for new subclasses of Ma-Minda bi-univalent functions," Journal of Inequalities and Applications, vol. 2013, article 317, 2013.

[5] R. M. El-Ashwah, "Subclasses of bi-univalent functions defined by convolution," Journal of the Egyptian Mathematical Society, vol. 22, no. 3, pp. 348-351, 2014. 
[6] B. A. Frasin and M. K. Aouf, "New subclasses of bi-univalent functions," Applied Mathematics Letters, vol. 24, no. 9, pp. 15691573, 2011.

[7] M. K. Aouf, R. M. El-Ashwah, and A. M. Abd-Eltawab, "New subclasses of biunivalent functions involving Dziok-Srivastava operator," ISRN Mathematical Analysis, vol. 2013, Article ID 387178, 5 pages, 2013.

[8] S. Bulut, "Coefficient estimates for a class of analytic and biunivalent functions," Novi Sad Journal of Mathematics, vol. 43, no. 2, pp. 59-65, 2013.

[9] S. Bulut, "Coefficient estimates for new subclasses of analytic and bi-univalent functions defined by Al-Oboudi differential operator," Journal of Function Spaces and Applications, vol. 2013, Article ID 181932, 7 pages, 2013.

[10] M. Çağlar, H. Orhan, and N. Yağmur, "Coefficient bounds for new subclasses of bi-univalent functions," Filomat, vol. 27, no. 7, pp. 1165-1171, 2013.

[11] T. Hayami and S. Owa, "Coefficient bounds for bi-univalent functions," Panamerican Mathematical Journal, vol. 22, no. 4, pp. 15-26, 2012.

[12] S. Porwal and M. Darus, "On a new subclass of bi-univalent functions," Journal of the Egyptian Mathematical Society, vol. 21, no. 3, pp. 190-193, 2013.

[13] H. M. Srivastava, S. Bulut, M. Çağlar, and N. Yağmur, "Coefficient estimates for a general subclass of analytic and biunivalent functions," Filomat, vol. 27, no. 5, pp. 831-842, 2013.

[14] Q.-H. Xu, Y.-C. Gui, and H. M. Srivastava, "Coefficient estimates for a certain subclass of analytic and bi-univalent functions," Applied Mathematics Letters, vol. 25, no. 6, pp. 990-994, 2012.

[15] Q.-H. Xu, H.-G. Xiao, and H. M. Srivastava, "A certain general subclass of analytic and bi-univalent functions and associated coefficient estimate problems," Applied Mathematics and Computation, vol. 218, no. 23, pp. 11461-11465, 2012.

[16] R. M. Ali, S. K. Lee, V. Ravichandran, and S. Supramaniam, "Coefficient estimates for bi-univalent Ma-Minda starlike and convex functions," Applied Mathematics Letters, vol. 25, no. 3, pp. 344-351, 2012.

[17] S. S. Kumar, V. Kumar, and V. Ravichandran, "Estimates for the initial coefficients of bi-univalent functions," http://arxiv.org/ $\mathrm{abs} / 1203.5480$.

[18] C. Pommerenke, Univalent Functions, Vandenhoeck and Rupercht, Göttingen, Germany, 1975. 


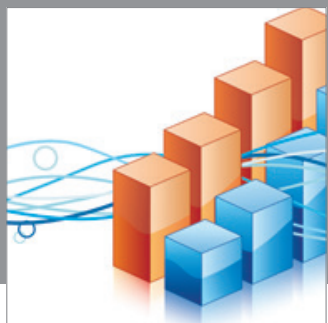

Advances in

Operations Research

mansans

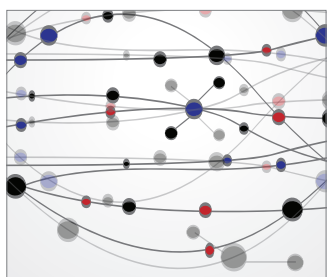

The Scientific World Journal
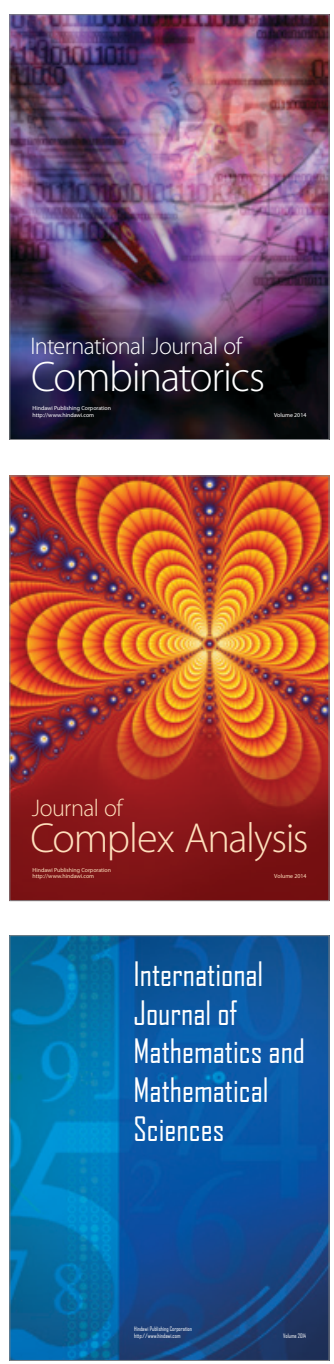
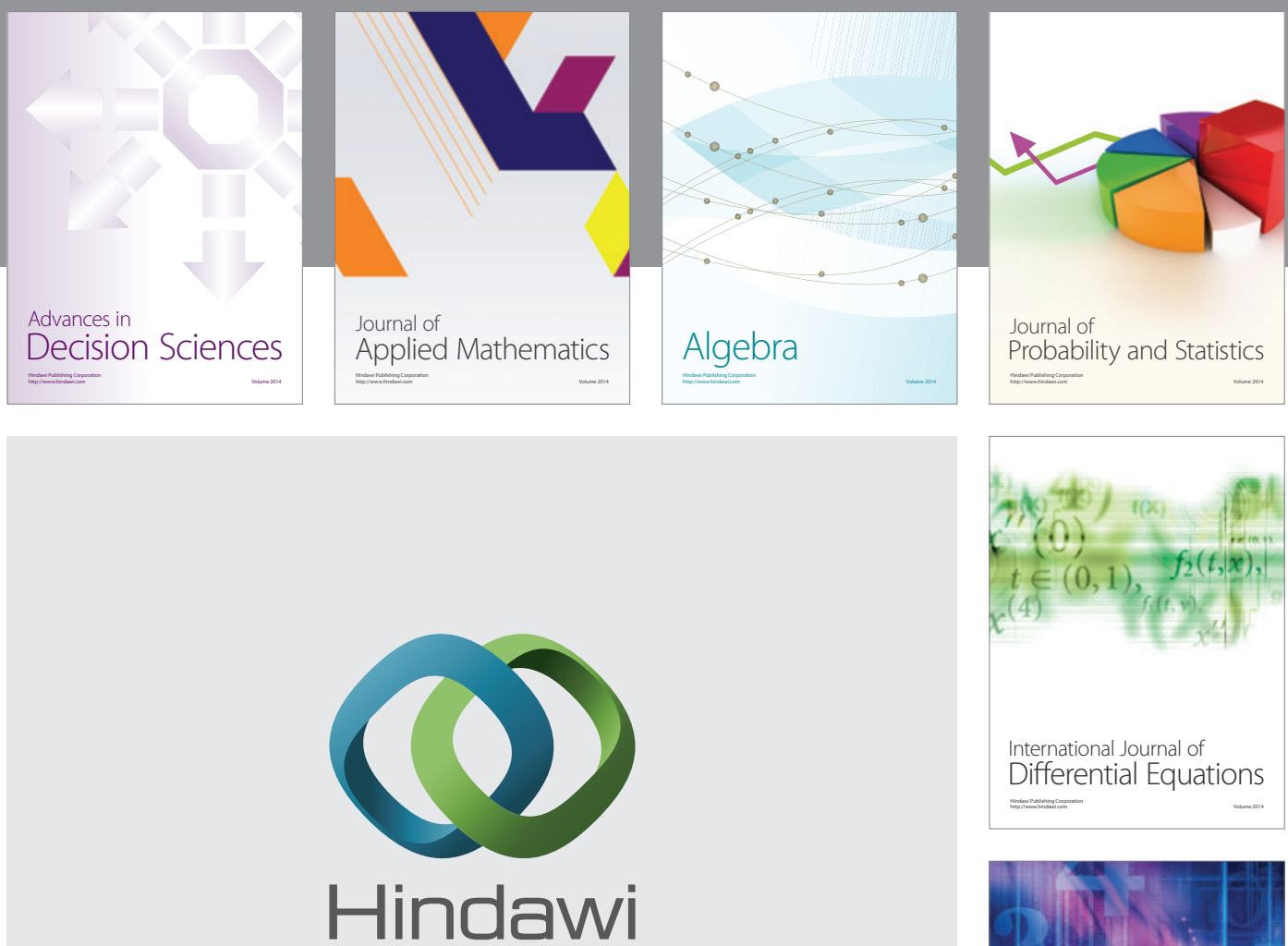

Submit your manuscripts at http://www.hindawi.com
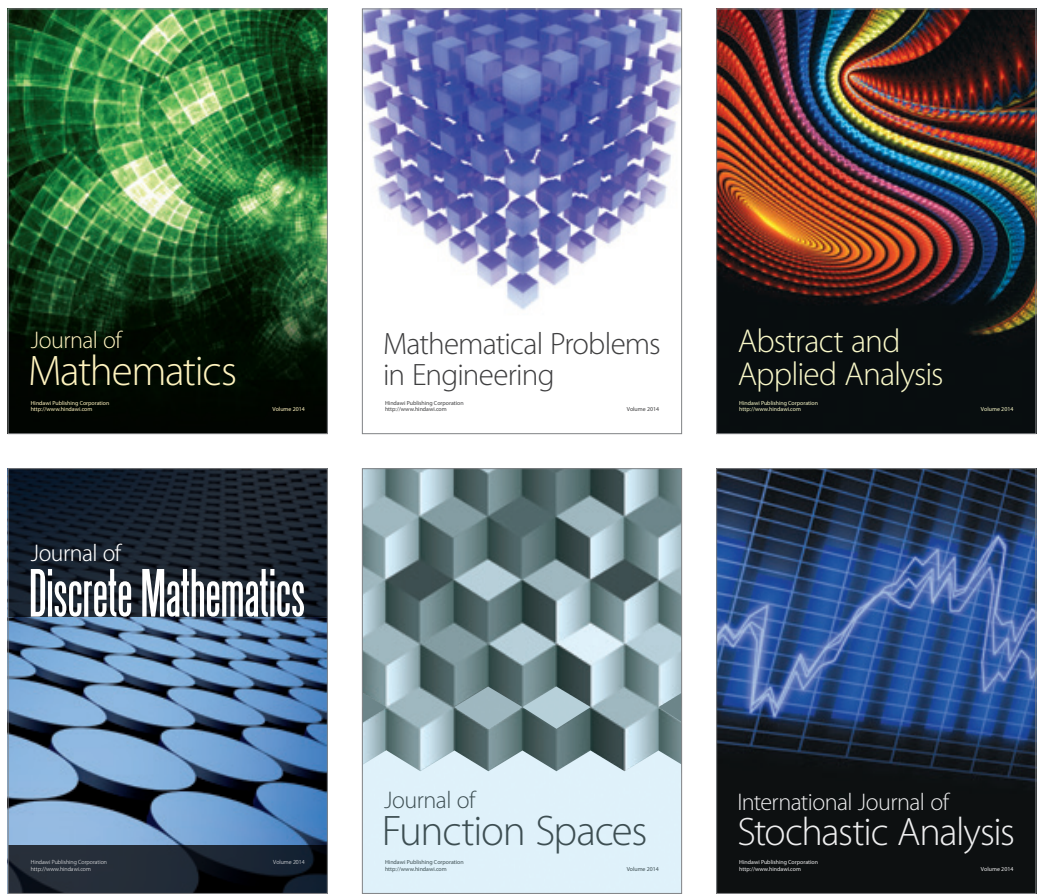

Journal of

Function Spaces

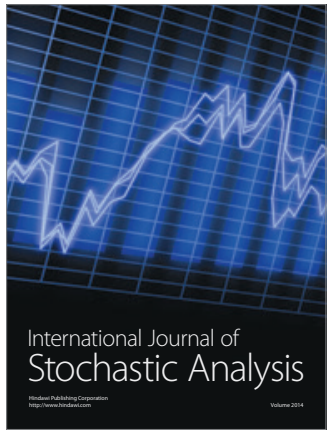

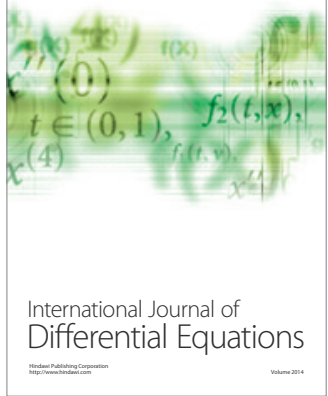
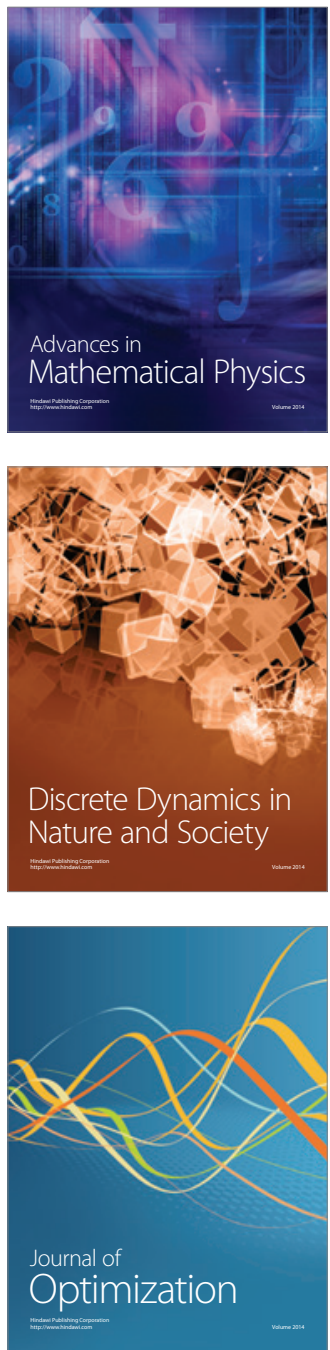